\title{
The effect of crosstalk on depth magnitude in thin structures
}

\author{
Inna Tsirlin*, Laurie M. Wilcox and Robert S. Allison \\ Centre for Vision Research, York University, 4700 Keele st., Toronto, ON, Canada
}

\begin{abstract}
Stereoscopic displays must present separate images to the viewer's left and right eyes. Crosstalk is the unwanted contamination of one eye's image from the image of the other eye. It has been shown to cause distortions, reduce image quality and visual comfort and increase perceived workload when performing visual tasks. Crosstalk also affects one's ability to perceive stereoscopic depth although little consideration has been given to the perception of depth magnitude in the presence of crosstalk. In this paper we extend a previous study (Tsirlin, Allison \& Wilcox, 2010, submitted) on the perception of depth magnitude in stereoscopic occluding and non-occluding surfaces to the special case of crosstalk in thin structures. Crosstalk in thin structures differs qualitatively from that in larger objects due to the separation of the ghost and real images and thus theoretically could have distinct perceptual consequences. To address this question we used a psychophysical paradigm, where observers estimated the perceived depth difference between two thin vertical bars using a measurement scale. Our data show that crosstalk degrades perceived depth. As crosstalk levels increased the magnitude of perceived depth decreased, especially for stimuli with larger relative disparities. In contrast to the effect of crosstalk on depth magnitude in larger objects, in thin structures, a significant detrimental effect was found at all disparities. Our findings, when considered with the other perceptual consequences of crosstalk, suggest that its presence in S3D media even in modest amounts will reduce observers' satisfaction.
\end{abstract}

Keywords: Crosstalk, ghosting, 3D displays, perceived depth, psychophysics, depth magnitude, thin structures

\section{INTRODUCTION}

Crosstalk in a stereoscopic display refers to the incomplete segregation of the two eyes' images. Ghost images, or ghosting, are the perceptual consequence of crosstalk. Virtually all popular commercial stereoscopic display systems are affected by crosstalk to varying degrees (see ${ }^{1}$ for a review). Unfortunately comparison of the exact levels of crosstalk in various systems is difficult since crosstalk measurement depends not only on the particular system components but also on the measurement method employed. In general, anaglyph systems have the most crosstalk while time-sequential displays and polarized displays exhibit the least amounts of crosstalk ${ }^{2,3}$.

More is known about the perceptual effects of crosstalk in stereoscopic viewing. Seuntiens et $\mathrm{al}^{4}$ reported that the amount of perceived distortion (ghosting, double-lines) increases with increasing crosstalk. Another study ${ }^{5}$ showed that $75 \%$ of observers chose crosstalk as the most important attribute in determining image quality. Accordingly, quality ratings of S3D images in that study decreased with increase in crosstalk. Pala et al. ${ }^{6}$ found that perceived workload was increased in the presence of crosstalk. In addition, several studies have reported that viewing comfort was reduced as crosstalk was increased ${ }^{7-9}$ particularly for images containing large disparities ${ }^{7}$.

Crosstalk has also been found to affect depth perception of S3D stimuli. In one study the ability to discriminate the convexity/concavity of a 3D sphere and to align two rods in depth was hindered by the presence of ghosting ${ }^{6}$. In another study, when observers judged depth in natural and artificial images using a Likert-like scale ${ }^{10}$, it was found that increase in crosstalk resulted in degraded depth quality (but see Seuntiens et al. ${ }^{4}$ ).

These studies considered qualitative/categorical depth perception or the ability to discriminate very small depth intervals. In many stereoscopic displays, disparities are usually well above perceptual threshold and it is arguable that the perception of depth magnitude, space and volume should be of principal concern. In two recent experiments we have assessed the effect of crosstalk on perceived depth magnitude from binocular disparity and monocular occlusions using a rigorous quantitative method (Tsirlin et al., 2010 submitted). We asked our observers to indicate the magnitude of depth in the stimuli using a (virtual) ruler while systematically varying the amount of simulated crosstalk and the disparity (in disparity based stimuli). In both cases we found an adverse effect of crosstalk on perceived depth; as crosstalk increased, perceived depth decreased. In the stimuli where depth was based on binocular disparity we used wide bars such that the ghost image always overlapped with the original stimulus lines. The goal was to examine the effect of crosstalk on large objects for which the ghost image rarely separates from the

* itsirlin@yorku.ca; phone 1416 736-2100 x 70430; http://www.wilcoxlab.yorku.ca/ inna 
original. However, these results may not generalize to S3D stimuli in which the ghost image is laterally separated from the real image of the object. This will occur when thin structures such as tree branches, wire fences or cords are presented with even moderate disparities. Note that thin here refers to the projected width of the object relative to its disparity - the segregation of the ghost image will also occur for large elongated objects presented at large disparities.

A

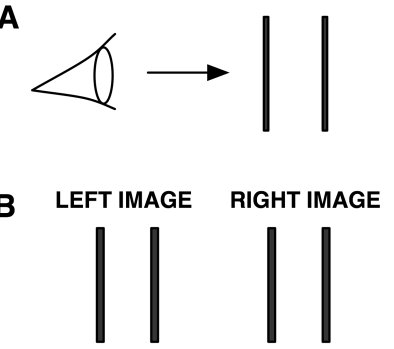

C

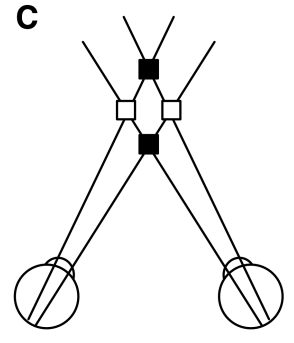

Figure 1 The double-nail illusion. (A) An observer views two thin objects located one behind the other in depth. (B) The projection of the objects on the retina. (C) A diagram (top view) that shows the possible matching solutions. The two objects can either be perceived one behind the other (filled squares) or side-by-side (empty squares), both arrangements produce the same retinal projection shown in (B). Observers normally perceive the two objects side-by-side.

This situation is qualitatively different than that where the ghost and the real image overlap. With thin structures the ghost and the real image are perceptually (and physically) separate. Different patterns of matching can be applied to the left and the right images since now instead of one object (albeit composed of two overlapping surfaces) we have two separate objects in each eye, the original and the ghost. Even for the simple case of a single object this leads to ambiguous matching. This situation is analogous to the 'double nail illusion' ${ }^{11}$ where the stereoscopic projection of two thin nails placed one behind the other is equivalent to the projection of two nails side by side as shown in Figure 1. Moreover, in the case of relatively wide objects at small disparities there is an alternative interpretation of the ghost image as edge blur or as a self-occlusion. These interpretations are not viable in the case of thin structures with separated ghost images. Thus we hypothesize that there will be a stronger effect of ghosting on perceived depth magnitude in thin structures with small disparities than would be seen when using wide objects. We used a depth estimation method similar to that employed in our previous experiments (Tsirlin et al., 2010 submitted). Thin structures were simulated as a pair of thin lines and the range of disparities was carefully chosen such that for all disparities the ghost and the original object did not overlap. We found that as crosstalk increased perceived depth decreased. The effect was similar to that observed in our previous experiment (in which the ghost images were not separated from the source image). However, the effect of crosstalk at the smallest test disparity was found only in the thin line configuration where the source and ghost images were separated.

\section{METHODOLOGY}

\subsection{Observers}

Nine observers, two authors (IT and LW) and seven volunteers (graduate and undergraduate students), participated in the study. All observers had normal or corrected-to-normal visual acuity and good stereoacuity (able to discriminate disparities at least as small as 40 seconds of arc). The interocular distance for each observer was measured with a Richter digital pupil distance meter.

\subsection{Apparatus}

The stimuli were presented using the Psychtoolbox (v. 7.0.8) package for MATLAB (v. 7.4) executed on a G5 Power Macintosh. Stimuli were viewed on a pair of CRT monitors (ViewSonic G225f) arranged as a mirror stereoscope (see Figure 2). The viewing distance was $0.6 \mathrm{~m}$, the resolution of the monitors was $1280 \mathrm{x} 960$ pixels and the refresh rate was $75 \mathrm{~Hz}$.With these settings each pixel subtended 1.77 minutes of visual angle. Gamma correction was employed to linearize the monitors. A chin rest was used to stabilize observers' head position during testing. 


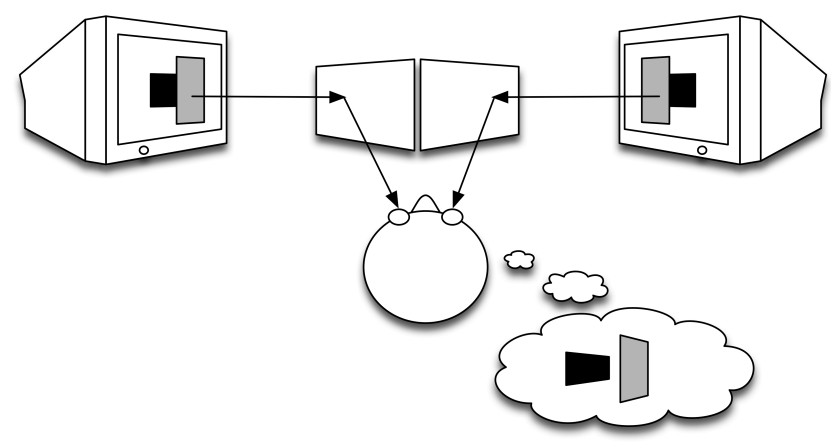

Figure 2 Mirror stereoscope. The left and right eyes' images are presented on two CRT displays. The images are then reflected from two mirrors into the observer's eyes. This type of spatial multiplexing provides crosstalk free stereoscopic images.

\subsection{Stimulus}

The stimulus consisted of two vertical lines of size 1.77 x 177 arc min, which were positioned around the midline separted by $88.5 \mathrm{arc}$ min. The left line had an uncrossed and the right line an equal crossed disparity of 3.54, 7.08, $10.62,14.16$, or 17.7 arc min with respect to the plane of the display (the total disparity between the lines was 7.08 , $14.16,21.24,28.32$ or 35.4 arc min respectively). The width of the stimulus lines was chosen carefully so that at all disparities the ghost images of the lines would not overlap with the real lines (at the smallest disparity the ghost image was just touching the stimulus line).

Angular disparities were converted to theoretical depth in centimeters in the Results and Discussion sections, to simplify the comparison with perceived depth. We used a standard formula, which relates disparity to theoretical depth at a known distance (see ${ }^{12}$ pp. 4-5). In the computations we used the average interocular distance of our observers $(6.07 \mathrm{~cm})$. The depths of each line relative to the screen corresponding to disparities of 3.54, 7.08, 10.62, 14.16 and 17.7 arc min were $0.61,1.22,1.83,2.44$ and $3.06 \mathrm{~cm}$ respectively (the total depth between the two lines was $1.22,2.44,3.67,4.89$ and $6.11 \mathrm{~cm}$ respectively).

A fixation cross $(26.5 \times 26.5$ arc min) was positioned 53.1 arc min above the stimulus. The vertical lines of the cross were presented as a Nonius line pair. That is, one line was presented only to one eye and the other line only to the fellow eye. When the observer's eyes are converged correctly the vertical lines appear aligned; if the eyes are misconverged the markers will be laterally displaced from one another.

A vertical ruler with an adjustable cursor was positioned 70.8 arc min below the stimulus. The ruler was 354 arc min long and the cursor was 7.08 arc min wide. The cursor could be moved along the ruler using a computer mouse. The elements of the display along with examples of the stimuli are shown in Figure 3.

The screen background was black and all the other elements were light gray (grayscale 193, luminance $78.95 \mathrm{~cd} / \mathrm{m}^{2}$ ). Crosstalk was simulated by adding an attenuated version (one of $0,1,2,4,8,16$ or 32\%) of the right image to the left image and vice versa. The corresponding gray levels of the ghosts were $0,1.9,3.9,7.7,15.4,31.0$ and 62.0 . To ensure that the color resolution of our displays was fine enough to represent each of the chosen gray levels we measured the corresponding luminance for each level using a photometer (10 measurements per level). The luminance was significantly different for all of the gray levels on both of the monitors (luminance $0,0.63,1.32,2.70$, $5.81,11.94,25.54 \mathrm{~cd} / \mathrm{m}^{2}$ ). We also presented the gray levels on the monitors and asked a subset of our observers to detect the change in luminance for consecutive gray levels. Observers indicated that consecutive pairs of gray levels were distinguishable. 


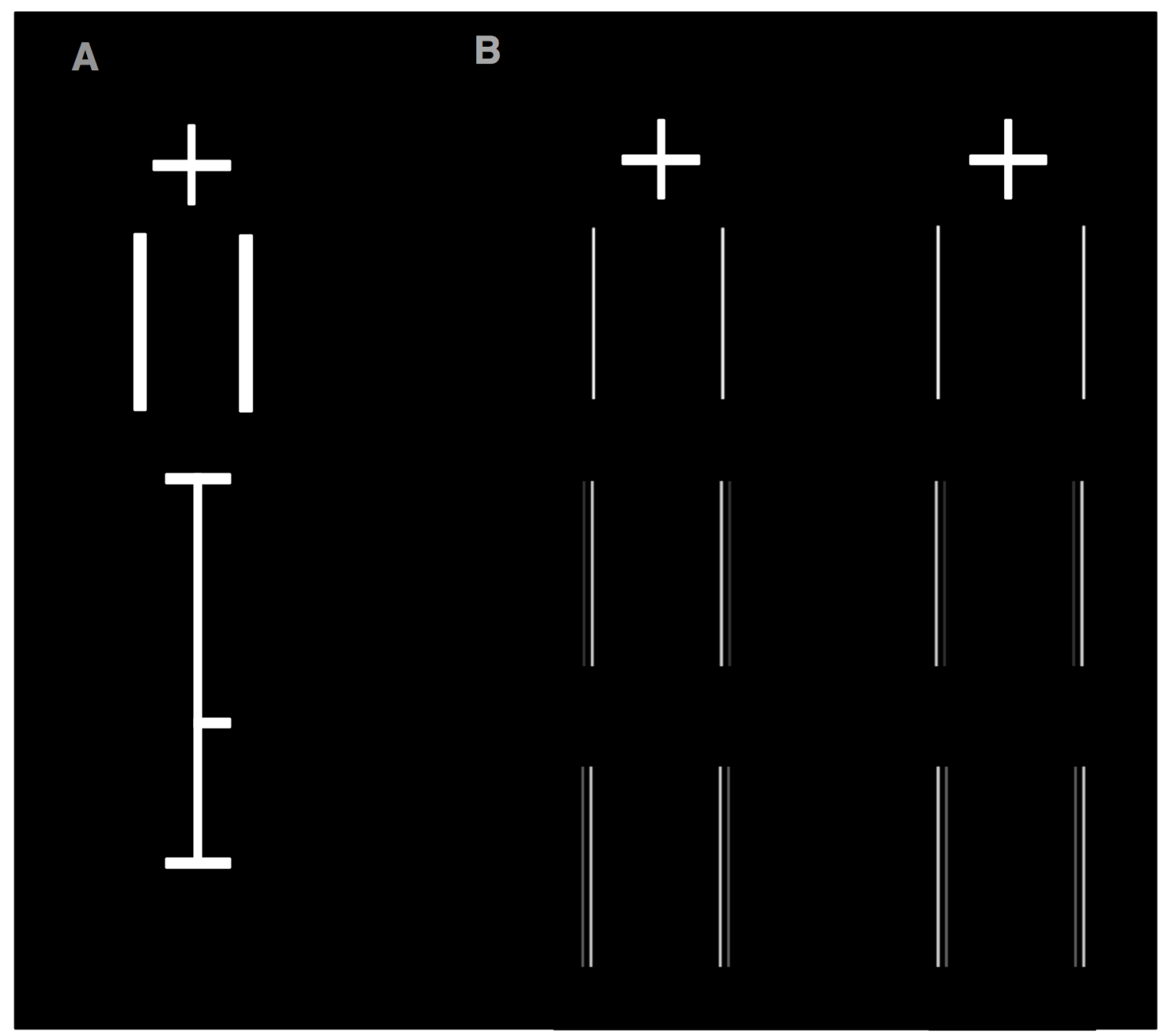

Figure 3 Stimuli. (A) Schematic of the complete display. (B) Example of stimuli arranged for free fusing. On the top row there is no crosstalk, the middle and bottom rows have $16 \%$ and $32 \%$ crosstalk accordingly. The lines have a disparity of $10.62 \mathrm{arc} \min .(1.83 \mathrm{~cm})$ with respect to fixation.

\subsection{Procedure}

Observers adjusted the sliding cursor on the ruler to indicate the amount of depth they perceived between the two stimulus lines. All estimates were made relative to the base/bottom of the scale. Although observers were free to move their eyes, they were encouraged to use the fixation cross to stabilize their gaze throughout a trial. Each of the 35 conditions ( 7 crosstalk levels x 5 disparities) was presented 10 times in random order in two sessions of 175 trials each. The experiment took place in a completely dark room.

\section{RESULTS}

Mean data for all observers are shown in Figure 4. The leftmost graph shows perceived depth magnitude as a function of crosstalk. Individual lines indicate data for different depth intervals, defined in terms of theoretical disparity-specified depth. In the absence of an effect of crosstalk, all lines would be parallel to the $\mathrm{x}$-axis, but this is clearly not the case. Instead, as crosstalk increases there is a decrease in perceived depth at all disparities. This effect can be appreciated from a different perspective in the right-hand graph of Figure 4. Here we replotted perceived depth as a function of the disparity-predicted depth in $\mathrm{cm}$, now each line corresponds to a different level of crosstalk. If crosstalk had no effect then the lines on this graph would coincide. It is clear that for large disparities, perceived depth was reduced at crosstalk levels as low as $4 \%$. 
Note that the estimated depth in the base condition with $0 \%$ crosstalk was lower than the theoretical depth we computed for each disparity. This underestimation could have been caused by the observers' underestimation of the viewing distance. Perceived depth from the same amount of disparity scales with viewing distance. The shorter the viewing distance the smaller perceived depth between two objects will appear given the relative disparity is kept constant. Underestimation of viewing distance can easily occur in a completely dark room where vergence and accommodation serve as the only cues to absolute distance (for review see ${ }^{12}$ sections 24.5 and 24.6).
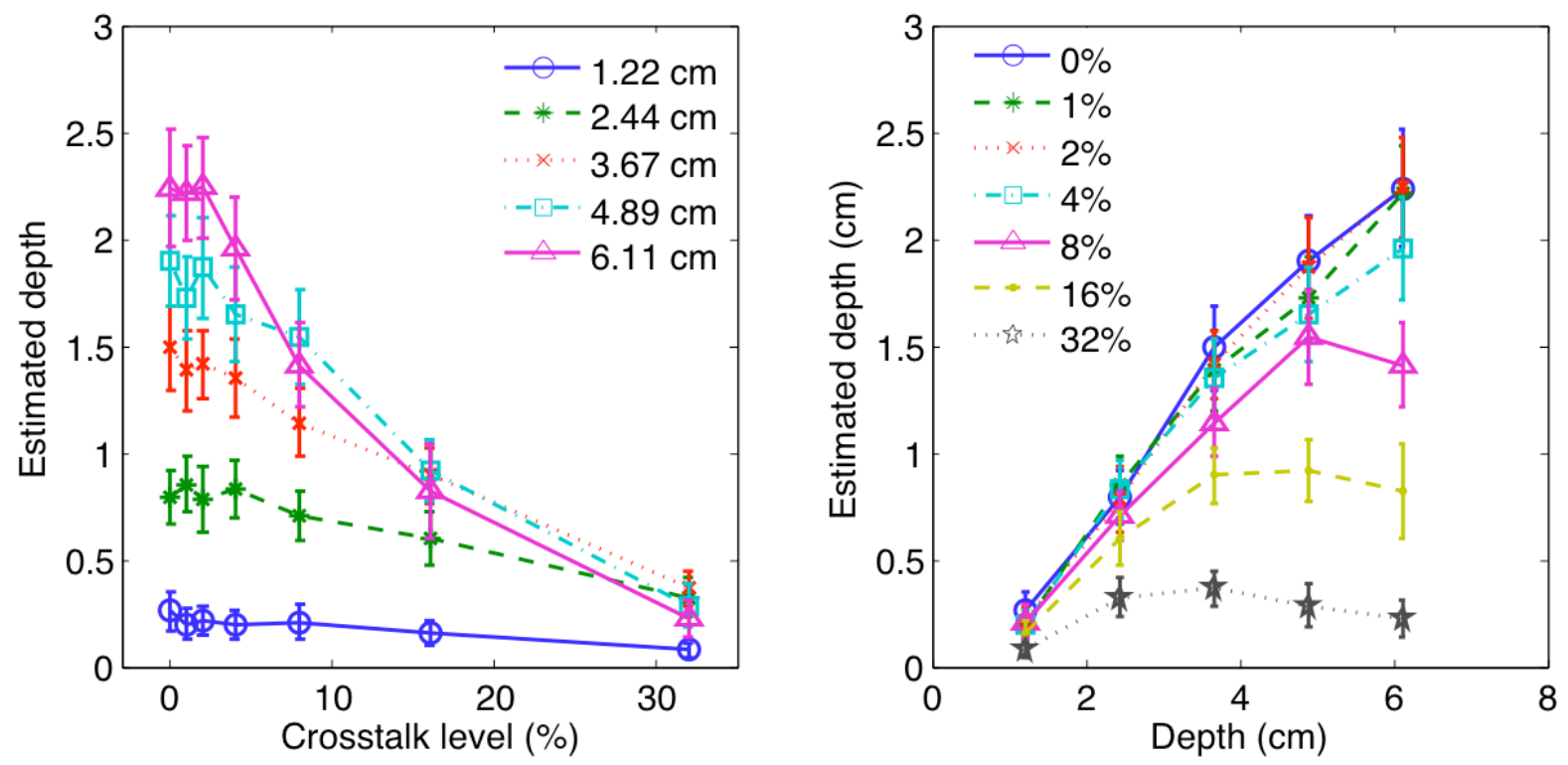

Figure 4 The mean data for the nine observers. Left panel: the abscissa shows the crosstalk levels and the ordinate the depth estimates. The different lines show stimuli with different disparities. The disparities are expressed in terms of the corresponding theoretical depth (see text). Right panel: the abscissa shows the theoretical depth corresponding to the different disparities and the ordinate shows the depth estimates. The colored lines show the stimuli with different crosstalk levels. The error bars indicate $+/-1$ standard error.

Since there was a relatively large difference between the perceived depth of the largest and the smallest disparities, the magnitude of the effect of crosstalk at the smallest disparities might not be appreciable in Figure 4. To examine the effects in the small disparity range more closely we normalized the data for each disparity for each observer by dividing the depth estimates for each disparity by the largest estimate obtained for that disparity. The averaged normalized data are depicted in Figure 5. It can be seen in this figure that depth judgments at all disparities were affected by crosstalk. Perceived depth magnitude was substantially reduced at as little as $4 \%-8 \%$ crosstalk. Even at the smallest disparity we can see a large effect of crosstalk, however, crosstalk seems more disruptive for larger disparities.

These observations were confirmed by statistical analysis. To analyze the data we used a nonparametric Wilcoxon signed-rank test. To establish at which level of crosstalk the estimated depth became significantly reduced we compared each of the non-zero crosstalk conditions to the zero crosstalk condition using paired tests. We conducted this analysis for each disparity separately. All statistical analyses used alpha level of 5\% and a one-tailed test and were performed using the statistical software package R. The results of the analysis are summarized in Table I in Appendix A.

For the three largest disparities, corresponding to depths of $3.67,4.89$ and $6.11 \mathrm{~cm}$, perceived depth was significantly reduced (relative to the $0 \%$ crosstalk baseline) for all crosstalk levels equal to or larger than $4 \%$. For disparity corresponding to depth of $4.89 \mathrm{~cm}$ there was also a significant difference between $0 \%$ and $1 \%$ of crosstalk, however, this result might be spurious since there was no significant difference between $0 \%$ and $2 \%$ crosstalk conditions for this disparity. For the two smallest disparities (corresponding to 1.22 and $2.44 \mathrm{~cm}$ ) depth was significantly reduced for all crosstalk levels equal to or larger than $8 \%$. For the smallest disparity there was also a significant decrease between $0 \%$ and $1 \%$, but a significant increase in depth between $0 \%$ and $2 \%$ crosstalk and no significant difference between $0 \%$ and $4 \%$ crosstalk. Due to this inconsistency and the high response variability at this disparity (see Figure 5) we consider the significant reduction of depth in this condition to start at $8 \%$. 


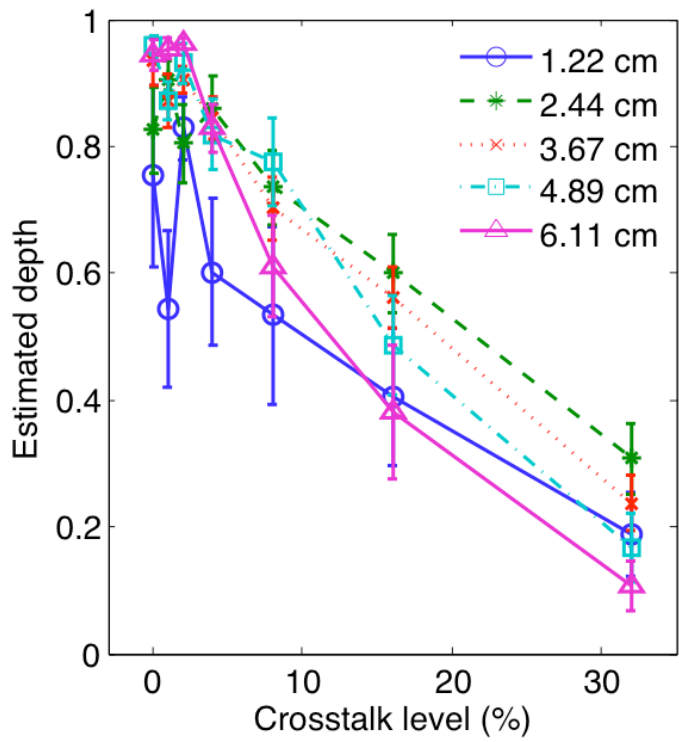

Figure 5 Results with data normalized per disparity. The abscissa shows the crosstalk levels and the ordinate the normalized depth estimates. The different lines show the stimuli with different disparities. The disparities are expressed in terms of the corresponding theoretical depth (see text). The error bars indicate $+/-1$ standard error.

The decline in perceived depth expressed as a percentage $(100 \%$ - (depth at $\mathrm{n} \%$ crosstalk / depth at $0 \%$ crosstalk)) tended to increase with increasing disparity (see Table I). For example, the reduction in perceived depth in comparison to the base line at $32 \%$ crosstalk was generally larger for larger disparities $(70,59,75,85$ and $90 \%$ for disparities corresponding to depths $1.22,2.44,3.67,4.89$ and $6.11 \mathrm{~cm}$ ).

We also computed the rate of change in perceived depth using the slope of the line between each two consecutive crosstalk levels $(0-1 \%, 1-2 \%, 2-4 \%$ etc.). We have plotted the mean slope for each disparity in Figure 6 . Mean slopes were computed using normalized data (see above) by taking only the slopes corresponding to statistically significant differences between two consecutive crosstalk levels. As can be seen in the figure, mean slope generally increases with increasing disparity. Taken together the percent decrease in perceived depth, the mean slopes and the smaller crosstalk levels at which perceived depth is significantly reduced indicate that larger disparities are more affected by crosstalk than the smaller disparities.

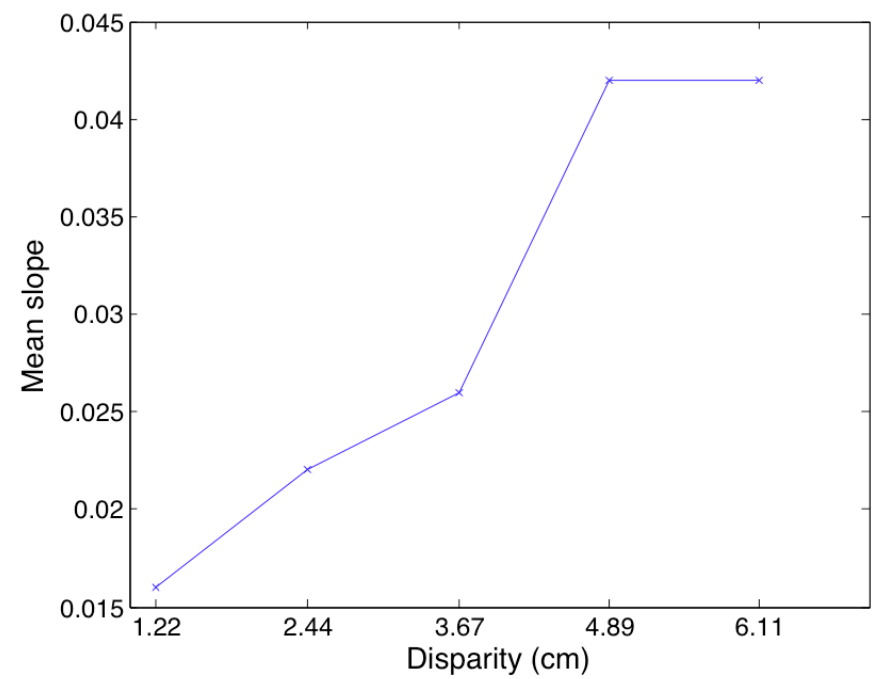

Figure 6 Mean slopes (measure of rate of change in perceived depth per unit change in crosstalk). The abscissa shows the different stimulus disparities. The disparities are expressed in terms of the corresponding theoretical depth. The ordinate shows the mean slope. See text for details. 


\section{DISCUSSION}

In the present experiment we found a detrimental effect of crosstalk on perceived depth magnitude in thin structures. As the amount of crosstalk in the stimulus increased, the magnitude of perceived depth decreased relative to the $0 \%$ crosstalk baseline condition. This effect was more pronounced for displays with larger relative disparities between the stimulus lines in terms of both absolute and proportional depth.

The arrangement of each of the two lines and their ghost images in our stimulus is analogous to the 'double-nail' arrangement ${ }^{11}$ shown in Figure 1. When the 'double-nail' stimulus is viewed stereoscopically, the thin objects (nails) are often seen positioned side-by-side at the same depth, although, in reality they are placed one behind the other in depth. This occurs since the retinal projection of the real arrangement is identical to the projection of the side-by-side arrangement. The projection of a thin object and its ghost, as in our experiment, is similar to that in the 'double-nail' projection (see Figure 7). Consequently, as in the classic illusion several matching solutions exist. In one, which we will refer to as 'in-depth', the originals are matched together and the ghosts are matched together, such that in the cyclopean view the original and the ghost will appear to be positioned one behind the other. In this case either the ghost or the original will be perceived as diplopic (double) due to the violation of the disparity gradient limit ${ }^{1}$. Thus, although the original is visible and its depth with respect to fixation can be estimated, diplopia can disrupt the percept of depth magnitude.
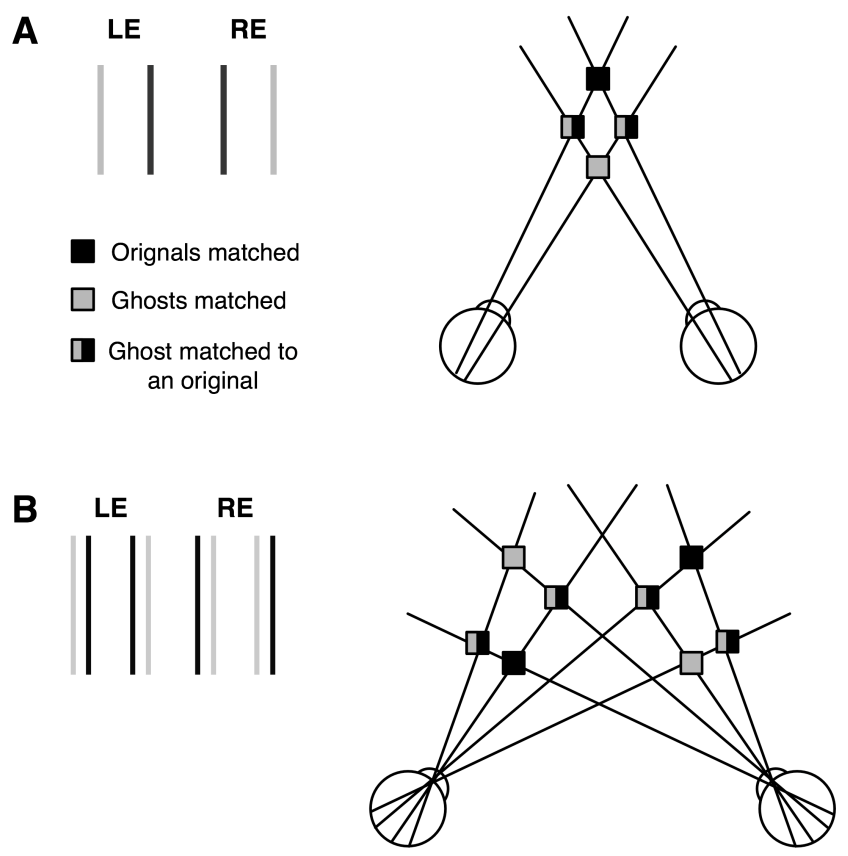

Figure 7 Matching solutions in our stimuli. In both panels (A) and (B) on the left are the half-images of the stimulus where black lines show the original and gray lines the ghost. On the right are diagrams (top view) that show the possible matching solutions; gray squares show matching of the ghosts, black squares show matching of the originals and black and gray squares show the matching of an original with a ghost. (A) For each line in our stimulus the arrangement is very similar to the 'double-nail' illusion arrangement (Figure 1). In this simple arrangement when the stimulus is located straight ahead in front of the eyes, the original and the ghost can either be perceived one behind the other, or as located side-by-side. We call these two solutions as 'in-depth' and 'side-by-side' respectively. (B) shows the stimulus used in our experiment. Here there are two original lines and their respective ghosts to the left and the right of fixation. One line has crossed and the other uncrossed disparity. In this case we have two 'double-nail' illusions and the matching arrangements are the same as in (A) although due to the central fixation in the 'sideby-side' case the lines might be seen in offset depth planes. As can be seen in the diagram, this depth offset depends on the ratio of the width of the complete stimulus to the interocular distance: the smaller the ratio, the smaller the offset. In our case this ratio is small $(1.62 / 6.07)$ hence we can assume that the offset 'side-by-side' case is negligible and the lines are seen as frontoparallel (phenomenology confirms this).

1 The disparity gradient of two objects is the ratio of their relative disparity to their angular separation. It has been shown that two objects cannot be simultaneously fused if the disparity gradient between them is larger than $1^{13}$. The disparity gradient between an object and its ghost (when the ghosts are matched together and the real objects are matched together) is equal to 2 . Consequently, it is not possible to fuse the stimulus lines and the ghost lines simultaneously. 
Alternatively, the ghost in one eye can be matched to the original located at the same retinal position in the other eye such that two copies of the original are perceived side-by-side at the same depth, just like in the 'double-nail' illusion. We refer to this matching solution as 'side-by-side'. In this case any depth the original had with respect to fixation will be largely eliminated (see Figure 7 caption for discussion of this condition in our case).

Thus both matching solutions would lead to a reduction of perceived depth of the original compared to a condition with no ghosting. However, in the 'side-by-side' case depth should be reduced more than in the 'in-depth' case.

Several factors seem to affect the way the matching ambiguity is resolved in our stimuli. In the canonical 'doublenail' illusion the two thin objects have the same luminance and contrast. In our case, the luminance (and contrast) of the original and the ghost are different. Smallman and $\mathrm{McKee}^{13}$ found that the matching of two features with different contrast depends on their contrast ratio. When the contrast ratio is within a certain range, matching will occur, while when the contrast ratio is outside of this range, the objects are not matched. The exact range depends on the contrast of the more luminous object. We have approximated this range in our experiment based on the data reported by Smallman and McKee and found that matching of the ghost with the original could occur for crosstalk levels larger than $13 \%$. However, this is only an approximation and additional experiments would be required to establish the exact range. It is possible that the switch from the 'in-depth' matching to 'side-by-side' matching at higher levels of crosstalk accounts for the larger reduction of depth at higher levels of crosstalk in our experiment, but this remains to be tested.

The choice of the matching solution could also be affected by the magnitude of the disparity of the original lines. When the disparity of the lines increases, the visual system - which has a bias to minimize the depth range in a scene $^{11}$ - might prefer 'side-by-side' matching in order to minimize the overall range of disparities. This would account for the stronger effect of crosstalk on thin line stimuli with larger disparities. The visual system could also alternate between the two matching solutions, perhaps with higher frequency in the larger disparity conditions yielding an unstable percept.

The present results are similar to the results of our previous experiment with wide objects. There, as in the current experiment, a decrease in perceived depth was observed with increase in crosstalk. This detrimental effect of crosstalk was also intensified with increasing disparity, however there is an important difference. In the previous experiment the ghost image always overlapped with the stimulus. As disparity increased the ghost image became more visible and thus more disruptive. At small disparities since the ghost was incorporated in the stimulus it could be perceived as blur around the edges of the object or as a contrast distortion. As a result, in the experiment with wide lines we found no effect of crosstalk on the smallest disparity. In contrast, in the present displays with thin objects, the ghost was visible even at small disparities and thus affected depth perception significantly even in stimuli with the smallest disparity. Based on these results we predict that stereoacuity, the smallest perceivable disparity, will be affected by crosstalk much more in thin objects than in wide objects. However, this remains to be investigated.

The present results and our previous experiments lead us to recommend a maximum crosstalk level in S3D displays of $4 \%$. At this level the perceived depth in disparity-based displays, both with wide and thin objects is reduced by about $7.6-9 \%$ on average and by $35 \%$ for monocular stimuli.

\section{APPENDIX A}

Table 1. Results of statistical analysis

\begin{tabular}{|l|l|l|l|l|l|}
\hline $\begin{array}{l}\text { Depth } \\
(\mathbf{c m})\end{array}$ & $\begin{array}{l}\text { Sample 1 } \\
\text { crosstalk } \\
(\mathbf{\%})\end{array}$ & $\begin{array}{l}\text { Sample 2 } \\
\text { crosstalk } \\
\mathbf{( \% )}\end{array}$ & p-value & $\begin{array}{l}\text { Diff. of } \\
\text { means } \\
(\mathbf{c m})\end{array}$ & $\begin{array}{l}\text { Reductio } \\
\mathbf{n}(\%)\end{array}$ \\
\hline \multirow{5}{*}{} & 0 & 1 & $0.018^{*}$ & 0.063 & 24.01 \\
\cline { 2 - 6 } & 0 & 2 & $0.027^{*}$ & 0.045 & 17.43 \\
\cline { 2 - 6 } & 0 & 4 & 0.092 & 0.062 & 23.88 \\
\cline { 2 - 6 } & 0 & 8 & $0.017^{*}$ & 0.052 & 19.92 \\
\cline { 2 - 6 } & 0 & 16 & $0.011^{*}$ & 0.105 & 40.11 \\
\cline { 2 - 6 } & 0 & 32 & $0.010^{*}$ & 0.182 & 69.85 \\
\hline
\end{tabular}




\begin{tabular}{|l|l|l|l|l|l|}
\hline \multirow{5}{*}{} & 0 & 1 & 0.875 & -0.058 & -7.34 \\
\cline { 2 - 6 } & 0 & 2 & 0.410 & 0.009 & 1.16 \\
\cline { 2 - 6 } & 0 & 4 & 0.820 & -0.036 & -4.47 \\
\cline { 2 - 6 } & 0 & 8 & $0.027^{*}$ & 0.089 & 11.14 \\
\cline { 2 - 6 } & 0 & 16 & $0.002^{*}$ & 0.191 & 24.02 \\
\cline { 2 - 6 } & 0 & 32 & $0.002^{*}$ & 0.468 & 58.86 \\
\hline \multirow{5}{*}{3.67} & 0 & 1 & 0.077 & 0.104 & 6.95 \\
\cline { 2 - 6 } & 0 & 2 & 0.125 & 0.077 & 5.16 \\
\cline { 2 - 6 } & 0 & 4 & $0.014^{*}$ & 0.137 & 9.17 \\
\cline { 2 - 6 } & 0 & 8 & $0.010^{*}$ & 0.350 & 23.45 \\
\cline { 2 - 6 } & 0 & 16 & $0.004^{*}$ & 0.596 & 39.96 \\
\hline \multirow{5}{*}{4.89} & 0 & 32 & $0.002^{*}$ & 1.123 & 75.37 \\
\cline { 2 - 6 } & 0 & 1 & $0.037^{*}$ & 0.172 & 9.10 \\
\cline { 2 - 6 } & 0 & 2 & 0.367 & 0.030 & 1.59 \\
\cline { 2 - 6 } & 0 & 4 & $0.040^{*}$ & 0.246 & 12.99 \\
\cline { 2 - 6 } & 0 & 0 & $0.014^{*}$ & 0.353 & 18.64 \\
\hline \multirow{5}{*}{6.11} & 0 & 32 & $0.002^{*}$ & 0.978 & 51.58 \\
\cline { 2 - 6 } & 0 & 1 & $0.002^{*}$ & 1.610 & 84.93 \\
\cline { 2 - 6 } & 0 & 2 & 0.326 & 0.022 & 0.98 \\
\cline { 2 - 6 } & 0 & 0 & 0.590 & -0.002 & -0.11 \\
\cline { 2 - 6 } & 0 & 16 & $0.006^{*}$ & 0.285 & 12.75 \\
\cline { 2 - 6 } & 0 & 32 & $0.002^{*}$ & 1.417 & 63.30 \\
\hline
\end{tabular}

\section{REFERENCES}

[1] Woods, A. J., "Understanding crosstalk in stereoscopic displays," Three-dimensional systems and applications. (2010).

[2] Pan, C. C., Lee, Y. R., Huang, K. F. et al., "Crosstalk evaluation of shutter-type stereoscopic 3D display," Society for information display 10. 128-131 (2010).

[3] Woods, A. J., Harris, C.R., "Comparing levels of crosstalk with red/cyan, blue/yellow, and green/magenta anaglyph 3D glasses," Stereoscopic displays and applications XXI. 7253, 0Q1-0Q12 (2010).

[4] Seuntiens, P. J. H., Meesters, L. M. J., and IJsselsteijn, W. A., "Perceptual attributes of crosstalk in 3D images," Displays, 26, 177-183 (2005).

[5] WIlcox, L. M., and Stewart, J. A. D., "Determinants of perceived image quality: ghosting vs. brightness " Stereoscopic Displays and Virtual Reality Systems X. 5006(2003).

[6] Pala, S., Stevens, R., and Surman, R., "Optical crosstalk and visual comfort of a stereoscopic display used in a real-time application," Stereoscopic displays and virtual reality systems XIV. 6490, 649011.1649011.12 (2007).

[7] Kooi, F., and Toet, A., "Visual confort of binocular and 3D displays," Displays, 25, 99-108 (2004).

[8] Lambooij, M., Fortuin, M. F., IJsselsteijn, W. A. et al., "Visual discomfort associated with 3D displays," Fifth International Workshop on Video Processing and Quality Metrics for Consumer Electronics 2010. (2010).

[9] Lambooij, M., and IJsselsteijn, W. A., "Visual discomfort and visual fatigue of stereoscopic displays: a review," Journal of Imaging Science and Technology, 53(3), 030201-1- 030201-14 (2009).

[10] Huang, K. C., Yuan, J. C., Hsueh, W. J. et al., "A study of how crosstalk affects stereopsis in stereoscopic displays," Stereoscopic displays and virtual reality systems X. 5006 247-253 (2003).

[11] Krol, J. D., and Grind, W. A. v. d., "The double-nail illusion: Experiments on binocular vision with nails, needles and pins," Perception, 9, 651-669 (1980).

[12] Howard, I. P., [Seeing in Depth] I. Porteous, Binocular fusion and rivalry (2002).

[13] Smallman, H. S., and McKee, S. P., "A contrast ratio constraint on stereo matching," Proceedings of the Royal Society of London, 260, 265-271 (1995). 